University of Wollongong

Research Online

Faculty of Social Sciences - Papers (Archive) Faculty of Arts, Social Sciences \& Humanities

2013

More thinking about less data: A perspective from the 2nd Provence

Summer Workshop

M Valenzuela

University of Sydney

D Bartres-Faz

University of Barcelona

E Bullmore

University of Cambridge

A Fjell

University of Oslo

M Maletic-Savetic

Baylor College Of Medicine

See next page for additional authors

Follow this and additional works at: https://ro.uow.edu.au/sspapers

Part of the Education Commons, and the Social and Behavioral Sciences Commons

Research Online is the open access institutional repository for the University of Wollongong. For further information contact the UOW Library: research-pubs@uow.edu.au 


\title{
More thinking about less data: A perspective from the 2nd Provence Summer Workshop
}

\author{
Abstract \\ Doppler intuited that a sound's pitch could be altered by the relative velocity between the source and an \\ observer-70 years later Hubble used the same principle and 42 data points to prove the universe was \\ indeed expanding. Arguably, no other data set of $0.042 \mathrm{~Kb}$ has done more to change our understanding of \\ the cosmos. Although modest in volume, it took Hubble several years to acquire these precious numbers.

\section{Keywords} \\ provence, 2nd, perspective, workshop, data, summer, less, about, thinking, more

\section{Disciplines} \\ Education | Social and Behavioral Sciences

\section{Publication Details} \\ Valenzuela, M., Bartres-Faz, D., Bullmore, E., Fjell, A., Maletic-Savetic, M., Martins, R., Solowij, N. \& Yucel, M. \\ (2013). More thinking about less data: A perspective from the 2nd Provence Summer Workshop. \\ Molecular Psychiatry, 18 (5), 524-525.

\section{Authors} \\ M Valenzuela, D Bartres-Faz, E Bullmore, A Fjell, M Maletic-Savetic, R Martins, N Solowij, and M Yucel
}


More thinking about less data: A perspective from the $2^{\text {nd }}$ Provence Summer Workshop.

Michael Valenzuela ${ }^{1 *}$, David Bartres-Faz ${ }^{2}$, Ed Bullmore ${ }^{3}$, Anders Fjell ${ }^{4}$, Mirjana MaleticSavetic ${ }^{5}$, Ralph Martins ${ }^{6}$, Nadia Solowij ${ }^{7}$, Murat Yücel ${ }^{8}$.

\section{Affiliations}

1. Regenerative Neuroscience Group, Brain and Mind Research Institute, University of Sydney, Australia

2. University of Barcelona, Spain

3. Cambridge University, United Kingdom

4. University of Oslo, Norway

5. Baylor College of Medicine, United States

6. Edith Cowan University, Australia

7. University of Wollongong, Australia

8. University of Melbourne, Australia

\section{Corresponding Author}

Associate Professor Michael Valenzuela

Regenerative Neuroscience Group

Brain and Mind Research Institute

University of Sydney

100 Mallett St Camperdown

NSW 2050

Australia 
Doppler intuited that a sound's pitch could be altered by the relative velocity between the source and an observer - 70 years later Hubble used the same principle and 42 data points to prove the universe was indeed expanding ${ }^{1}$. Arguably, no other dataset of $0.042 \mathrm{~Kb}$ has done more to change our understanding of the cosmos. Whilst modest in volume, it took Hubble several years to acquire these precious numbers.

Nowadays we conduct neuroscience in a state of instant data overload. In a matter of hours we could produce a structural image of an individual's brain comprising a matrix of 256 X 256 X $128=8,388,608$ data points, a resting-state functional MRI timeseries (83MB), and from a simple blood sample, derive the person's genetic sequence by GWAS $(30 \mathrm{~K})$, state of gene expression by microarray (another $30 \mathrm{~K}$ ) and metabolomic profile using any of a number of commercially available chips (1K). We needn't necessarily stop there. In principle, the number of brain-gene-omic interactions on permutation alone approaches $10^{19}$.

It's safe to predict that the ordinary person is not well placed to think about this uber matrix. Recall the human brain 'only' contains $10^{12}$ synapses. And even if we could literally combine brain power, it would take a cluster of 10,000,000 networked humans to allocate just a single synapse to any given interaction. Let's also ignore the fact that Matlab maxes out on a matrix greater than 8TB, or the pesky issue of Type I error.

Data reduction is therefore one of the key problems for a field trying to better understand the complexities of the human brain and how and why it sometimes breaks down. At the Workshop, we discussed two general counter-strategies. The first was data-driven. For example, computationally intense graph theoretical analyses fit within a wider systems biology framework that aims to reduce highly multi-dimensional data into less dimensional more tractable data. The second was having more time to think a great idea.

Graph theory searches for invariant structures within large matrices, patterns of covariance that we now appreciate are related to the ability of complex systems to transfer information. Ed Bullmore identified four areas where graph theory may help advance neuroscience ${ }^{2}$. Firstly, it provides novel metrics for looking at pre-existing data in a new way. Secondly, it helps compare and converge results across multiple image (and non-image) modalities. Thirdly, it may provide a tool for understanding how mechanistic changes at one level of enquiry relate to higher-order functional changes, as well as help translate findings from animal models to the human realm ${ }^{3}$. Finally, it may introduce new measures for use as outcomes in clinical trials, a topic of intense debate at the previous Workshop4.

On the other hand, the psychology and neuroscience of great thinking is virtually nonexistent 5 . Better insights come from literature and biography. Relativity is said to have occurred to Einstein as part of a process of mental simulation and imagination over a number of neighborhood walks. Proust began his Remembrances when the perfume of a madeleine over a lazy morning coffee triggered a wave of obsessional work.

Ordinary mortals also need time to think. If you're co-ordinating 16 projects, supervising 8 graduate students and drafting 6 papers simultaneously, it's unlikely you have much of it. Our Workshop under the warm Provencal sun is therefore designed to provide one of the necessary conditions for great thinking - unfettered time.

Whilst great thoughts can of course come anytime anyplace, the Workshop produced some contenders. Independently, two groups in Perth and Barcelona presented 
tantalizing new evidence about gene-environment interaction related to APOE4, otherwise considered a dementia and cardiovascular risk factor.

David Bartres-Faz showed that TMS applied to the prefontal cortex produces much more robust changes in functional connectivity in APOE4 positive elders than individuals without the E4 allele. Some of the resultant changes were an amplification of those associated with compensatory brain activity in successful ageing, and so may be of longitudinal benefit.

Ralph Martins presented how a general negative correlation between lifestyle-related physical activity and amyloid brain burden is much stronger in APOE4 carriers, suggesting that exercise may be a particularly effective intervention in those at most risk for incident dementia.

Suddenly APOE4 is seen in a new light. Perhaps not only is it a risk factor but also an opportunity to intervene more successfully. Perhaps we need to combine TMS and exercise as part of a prevention trial in only APOE4 positive elders?

Yet perhaps the neatest example of original thinking was provided by Anders Fjell who approached old data with fresh eyes. He took advantage of the ADNI dataset that is freely available to all researchers (http://adni.loni.ucla.edu). This wonderful resource means anyone with a decent computer can carry out a significant study on one of the world's largest brain MRI datasets at virtually no cost. Actually, not anyone. You need to know what to do with the data, and moreover, have an original idea.

Anders' concern was the thorny issue of the distinction between so-called normal ageing and Alzheimer's disease (AD) ${ }^{6}$. He had the clever idea to group older individuals into those with no evidence of long term cognitive decline or presence of AD biomarkers, typically understood to preclude the possibility of subclinical AD, versus various other phenotypes. He showed that longitudinal atrophy of the entorhinal cortex, one of the areas first targeted by AD, was detectable even in these 'super stable' cognitively-intact elders. Accelerated atrophy in those brain area often linked with AD does not uniquely signify neurodegenerative illness, but instead may also comprise 'normal aging' whatever that is.

To be clear there is nothing wrong with big data-hungry science. Indeed, there has in recent years been an astonishing growth in generation of truly enormous datasets. Yet one forceful message from the Workshop was that along with truckloads of data we need a commensurate clearing out of mental space. Great thoughts are ultimately the best type of data reduction procedure. 


\section{References}

1. Hubble E. A relation between distance and radial velocity among extragalactic nebulae. Proceedings of the National Academy of Sciences of the United States of America 1929; 15: 168-173.

2. Bullmore E, Sporns O. Complex brain networks: graph theoretical analysis of structural and functional systems (vol 10, pg 186, 2009). Nature Reviews Neuroscience 2009; 10(4).

3. Siette J, Westbrook RF, Cotman C, Sidhu K, Zhu W, Sachdev P et al. AgeSpecific Effects of Voluntary Exercise on Memory and the Older Brain. Biol Psychiatry 2012; epub ahead of print.

4. Valenzuela M, Bartres-Faz D, Beg F, Fornito A, Merlo-Pich E, Muller U et al. Neuroimaging as endpoints in clinical trials: are we there yet? Perspective from the first Provence workshop. Mol Psychiatry 2011; 16(11): 10641066.

5. Lee R. The Eureka! Moment. Routledge 2002.

6. Fjell A, Westlye L, Grydeland H, Amlien I, Espeseth T, et al. Accelerating cortical thinning: unique to dementia or universal in aging? Cerebral Cortex. epub ahead of print. 